\title{
Depression and Anxiety in Women after Childbirth During the COVID-19 Pandemic
}

\author{
Joanna Baran *, Ewelina Czenczek-Lewandowska, Justyna Leszczak, Aneta Weres and Rafał Baran Teresa Pop
}

Institute of Health Sciences, Medical College, University of Rzeszów, Al. mjr.W.Kopisto 2a, 35-310 Rzeszów, Poland; Natural and Medical Center for Innovative Research. University of Rzeszów, ul. Warzywna 1A, 35-310 Rzeszów Poland;

Introduction:

Regardless of whether a child is born by force of nature or by caesarean section, childbirth is a huge effort for the female body. We are talking about physical effort and pain, but also mental. A big dose of hormones that floods the female body makes itself felt within a few to several hours after childbirth. Sadness, loneliness, anxiety, fear are frequent emotions accompanying a woman after childbirth. In the time of the COVID-19 pandemic, all visits to hospitals, including gynecology and maternity units, are prohibited. The woman has to rely on herself and on the help of the medical staff. The aim of the study was to assess the occurrence of depression and anxiety disorders in postpartum women during the COVID-19 pandemic.

Material and methods:

The study group consisted of 130 women, 54 of whom gave birth during the pandemic (41.5\%, the study group). The age of the respondents ranged between 23-40 years. $80 \%$ of women had higher higher education. Their level of anxiety before and after childbirth was assessed using the General Anxiety Disorder-7 (GAD7) scale and the level of depression using the Edinburgh Postnatal Depression Scale (EPDS). In addition, asked o complications after childbirth, the severity of pain or the presence of mental disorders in the patient and in her family. The results were statistically processed in the Statistica 13 package.

Results:

The analysis showed that nearly half of the respondents had problems with lactation after childbirth (50.8\%) and pain that made impossible to take care of the baby $(46.15 \%$; $\mathrm{Me}=7$ ). Additionally, due to the COVID-19 pandemic, it was impossible to visit hospitals $(46.2 \%)$, which was particularly difficult for women after cesarean section (58.5\%). Most often it was the first (52.3\%) or second (40\%) delivery. In the case of anxiety, statistically significant differences before and after childbirth were obtained only in women who had 1 child at home $(\mathrm{p}=0.033)$, in women who experienced severe pain after delivery $(\mathrm{p}=$ $0.037)$ or had problems with lactation $(p=0.004)$. A more serious situation appeared in the case of depression symptoms, where statistically significant differences were found in almost every analyzed aspect. The most important ones include delivery during the pandemic ( $p<0.001)$, no visitation after delivery $(\mathrm{p}<0.001)$, lack of staff assistance $(\mathrm{p}<0.001)$, delivery by $C C(p<0.001)$, pain $(p<0.001)$ and problems with postpartum lactation $(p$ $<0.001)$.

Conclusions:

Childbirth is an enormous effort for a woman, both physically and mentally. At this particular time, a woman should be cared for and supported by medical staff and loved 
The 3rd International Electronic Conference on Environmental Research and Public Health

ones. Long-term depressive symptoms after childbirth may turn into permanent depression, which adversely affects the relationship between the mother and the child, as well as the child's development and recovery of the mother's strength and health before delivery. 